\title{
Tappetina: an Ecosystem of Art, Software, and Research
}

\author{
Letizia Jaccheri, Javier Gomez, Sindre B. Skaraas \\ Department of Computer Science, Norwegian Univeristy of Science and Technology. \\ (Trondheim) \\ \{letizia.jaccheri, javier.escribano\}@ntnu.no, sibsen@live.com
}

\begin{abstract}
Tappetina is a fairy tale about mentoring, social innovation and technology. A set of projects have been developed around the Tappetina concept. These include science workshops for teenagers and the associated material created by the participants, illustrations, a website, a video, a story telling game. These projects are all connected in an ecosystem of art, science, and research expressions.

Participants are supposed to experience the works sequentially. Participants will be invited into the projects and encouraged to give feedback about how they perceive the experience. Sessions for playing the collaborative story telling game will be organized.
\end{abstract}

Keywords: Fairy Tales, Serious Games, Affective Learning, Empathy, Collaborative Storytelling.

\section{Introduction}

We offer an as an artistic experience around an ecosystem of works. This ecosystem is formed under the novel of "The Little Doormaid: Tappetina", authored by Letizia Jaccheri. When asked why she wrote this story, Letizia answered that she hoped it could inspire girls who read it to be more interested in technology. The story would not only try to combat stereotypes of girls in tech, but also create an emotional impact that creates interest in and even romanticizes technological concepts. In essence, it aims to build enthusiasm in kids to tech, and is thus used in IT workshops for learning IT and programming. The ecosystem that came out of this is an initiative to encourage nuanced views of people. It is formed under to combat the stigma surround women entering male-associated fields such as technology.

The novel has inspired several actors who have cooperated to produce workshops, a video, and a story telling game. This work, when exhibited will offer an experience of at least 20 minutes if one chooses to participate to a gaming session.

All the materials related to the project and events can be found at Tappetina's webpage: www.tappetina.com.

The video is available at https://tinyurl.com/ybksf6dm. It has been developed by a group of students $\backslash$ and aims to encourage teenagers to study computer science and promote the contribution of women in computer science by advertising the female character "Tappetina". 


\section{About the authors}

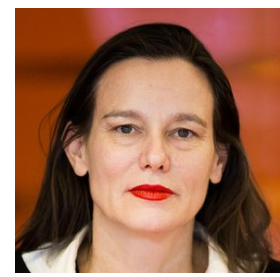

Letizia Jaccheri (Ph.D. from Politecnico di Torino, Italy) is Professor at the Depart- of Computer and Information Science.Jaccheri's research is on: software engineering; entertainment computing; computational creativity; ICT-enabled social innovation.
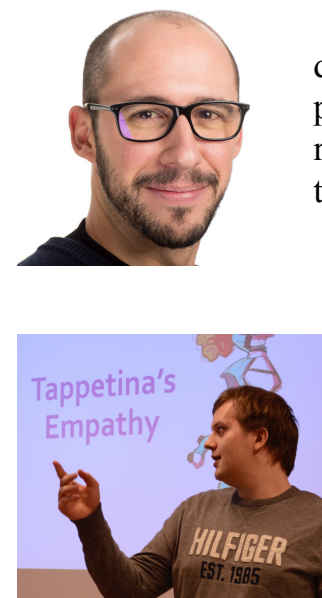

Javier Gomez (PhD. from Universidad Autónoma de Madrid, Spain) is an ERCIM "Alain Bensoussan" fellow at Department of Computer and Information Science, NTNU. His research interests include human-computer interaction, assistive technologies and serious games.

Sindre B. Skaraas graduated from NTNU in 2018 with a Master thesis titled Tappetina's Empathy A Study of Serious Games Facilitating Empathy with Storytelling.

\section{Acknowledgements}

This work has been partially supported by NTNU ARTEC and by the ERCIM fellowship program. The authors would like to thank Alexandra Angeletaki for organizing the international experiment workshop at the Gunnerus library of NTNU and all the participants to the Tappetina workshops. We thank the students who developed the video: Biljana Arsenic, Farzana Quayyum, Kshitiz Adhikari, Letizia Balzi, Md Shah Newaz, Nazli Sila Kara, and master student Uyen Dan Nguyen (Mimi) and PhD student Sofia Papavlasopoulou for workshop design and evaluation.

For the development of the game, the Unity Engine and editor was utilized, as developed by Unity Technologies. 